Archives of Anesthesiology

Volume 2, Issue 1, 2019, PP: 32-35

SRYYHWA

\title{
The Prediction of Difficult Airway: is it Possible?
}

\section{Ayten Saracoglu ${ }^{1 *}$, Kemal Tolga Saracoglu ${ }^{2}$}

\author{
${ }^{1}$ Department of Anesthesiology and Reanimation, Marmara University Pendik Training \\ and Research Hospital, Istanbul, Turkey. \\ ${ }^{2}$ Department of Anesthesiology and Reanimation, Health Sciences University Kartal Dr. Lutfi Kirdar Training \\ and Research Hospital, Istanbul, Turkey. \\ anesthesiayten@gmail.com
}

*Corressponding Author: Ayten Saracoglu, MD, DESA. Marmara Universitesi Hastanesi Fevzi Cakmak Mh. Muhsin Yazicioglu Cd. Pendik, Istanbul, Turkey.

\begin{abstract}
According to the 4th National Audit Project of the Royal College of Anaesthetists, the insufficient knowledge level, the familiarity with the situation, the presence of appropriate equipment, the existence of crisis management are the most important factors contributing to the existence. It is noteworthy that there is insufficient management plan or inadequate alternative plan development in the presence of these findings as well as inadequate airway evaluation of the anesthesiologist. Several studies have attempted to establish a scoring or mathematical model to provide predictions of difficult intubation. Despite all preoperative preparedness and predictive scoring systems, we have seen a relatively high rate of unexpectedly difficult airways in all patients despite having low airway risk score and with all normal examinations, and we have not yet determined reasons. In this review we aimed to analyse and compare the conventional airway assessment methods and new techniques.
\end{abstract}

Keywords: airway management; predictors; difficult airway; anesthesia.

\section{INTRODUCTION}

A common cause of morbidity and mortality associated with anesthesia is difficult or unsuccessful intubation. Difficult airway prediction is still a controversial issue for anesthesiologists in clinical practice. Because unexpected airway cases are seen in $25-30 \%$ of cases, this can be prevented by $10 \%$. According to 4 th National Audit Project of the Royal College of Anaesthetists (NAP4), in the practice of 114904 anesthesia from 309 hospitals between September 2008 and September 2009, the failure of the anesthesiologists in airway assessment has been identified as one of the biggest problems. Severe airway complications were seen in 184 patients, resulting in brain injury, urgent surgical airway requirement, unexpected hospitalization and 14 brain death. The insufficient knowledge level, the familiarity with the situation, the presence of appropriate equipment, the existence of crisis management are the most important factors contributing to the existence. It is noteworthy that there is insufficient management plan or inadequate alternative plan development in the presence of these findings as well as inadequate airway evaluation of the anesthesiologist (1). According to ASA Closed Claims, predicting difficult airway was found to be the most important factor leading to failed airway management. Inadequate ventilation was observed in $\% 12.7$ of the patients and in $\% 53$ of the patients.

The "cannot-intubate, cannot-oxygenate" condition is the most feared condition of anesthesiologists in clinical practice, otherwise it can be lost rapidly due to patient hypoxia (2). This situation is significantly similar to the risk analysis in the aviation sector and the resulting mortal results. Therefore, we should be able to give the answer to some questions while doing the anesthetic plan; Is there any clue about difficult airway prediction, can I ventilate the patient directly or indirectly, do you have difficulty in mask ventilation, and can I ventilate the patient with a face mask? Avoidance of complications associated with airway requires both individual and institutional preparation, including careful airway examination, a good anesthetic plan and back plan, good communication and teamwork, experience and knowledge, different devices and techniques. 
Many studies have attempted to establish a scoring or mathematical model to provide predictions of difficult intubation. Despite all preoperative preparedness and predictive scoring systems, we have seen a relatively high rate of unexpectedly difficult airways in all patients despite having low airway risk score and with all normal examinations, and we have not yet determined reasons. The method, test or imaging to be used for the optimasationof airwayevaluation is still a matter of debate. Therefore, the more diagnostic and validated tests are still under investigation. Airway history, detailed examination of head and neck, imaging techniques and some parameters and tests are used to determine difficult airway prediction. In the evaluation of airway, it is aimed to determine whether there is difficulty in the placement of supraglottic device, difficulty in intubation, ventilation. Airway compromise due to upper airway obstruction cause symptoms like stridor, hoarse voice, orthopnoea, drooling, dysphagia. Risk factorsare suspected as airway burn, neck trauma, infections of oropharynx and neck, previous surgery or radiotherapy to neck, problems with mouth opening e.g. trauma, soft tissue disorders, arthridities, problems with neck mobility e.g. cervical spine disruption, rheumatoid arthritis, cervical fusion (e.g. operative, ankylosing spondylitis, scleroderma), obesity, OSA, oropharyneal or neck masses, difficult dentition, pregnancy, recent intubation (swelling, trauma), angioedemai craniofacial syndromes, airway trauma (blunt or penetran).

\section{HisTORY}

Sniffing position was originally described by Chevalier Jackson as the "Boyce-Jackson" position in 1913. He published the first study of optimal patient positioning for orotracheal intubation, emphasizing the importance of anterior flexion of the lower cervical spine as well as the more obvious extension of the atlanto-occipital joint. Then the position was termed the sniffing position by Magill in 1936 as elevation of the occiput and extension of the head at the atlantooccipital joint. Bannister and MacBeth proposed the 3 axis alignment theory in 1944. Savva estimated the distance from the suprasternal notch to the mentum and inquired correlation with Mallampati score in 1948. Temporomandibular distance was termed by Patil in 1983 (3). These 4 grades of laryngoscopic views were defined by Cormack and Lehane in 1984. Horton et al. in 1989 to provide the angles of flexion of the lower neck and extension of the head. Levitan was descriebed POGO scores in 1998. Two curve theory was descriebed by Greenland 2008. Following years the upper lip bite test was proposed by Khan and Mallampati.

The improved availability of cone-beam computed tomography, 3-D imaging, and computer simulation has been used by Schendel and Hatcher for the evaluation of the airway.

Langeron et al. have previously assessed computerassisted modelsdeveloped a SCORE-computer with a predictive value. Mashour and Sandberg proposed that the MMP with extension (Extended Mallampati Score) in 2006. Kristensen et al. emphasized the linear high-frequency transducer to image superficial airway structures (within 2-3 cm from the skin) and that the curved low-frequency transducerfor explaining sagittal and parasagittal views of structures in the submandibular and supraglottic regions in 2013.

\section{INCIDENCE}

Difficult airway incidence varies between 0.08 and $15 \%$. Impossible mask ventilation incidence of $0.07 \%$. The incidence of difficult intubation is $6.2 \%$, the incidence of difficult ventilation and difficult intubation is $1.5 \%$, while the Can not intubate can not ventilate is seen in $0.07 \%$ (4). Another important point is that $94 \%$ of the difficult mask ventilation is unforeseen and $22 \%$ is very difficult.

\section{Two Curve Theory Three Column Model}

In fact, this model has a new concept in understanding the mechanism of laryngoscopy and an approach beyond the 3 axis alignment. Successful laryngoscopy requires the oropharyngeal curve and pharyngoglotto-tracheal curve. With the extention of the head, the primary curve is flattened, and for the secondary curve the flexion of the lower cervical spines is required with the head lift. According to the three axis theory; in the sniffing position, three axes are said to come on the same line, but because of atlantooccipital extension is limited, it is not possible to align the hard palate with the laryngeal and pharyngeal axes. Furthermore, a minimal change in the laryngeal axis occurs during atlanto-occipital extension when the pharyngeal axis changes to the posterior position. It is a structured airway evaluation which is divided into anterior, posterior and middle columns according to three column model. 
The Prediction of Difficult Airway: is it Possible?

In the analysis of the explored column, it is also possible to examine the separate plans:

1. Volume of submandibular space: It refers to the problems related to the elevation of the soft tissues. Measurement of three sides of the inverted triangle pyramid (TMJ-insulator, insulatorhyoid and TMJ-TMJ). A reduction in one or more of these distances leads to a small anatomical cavity by submandibular tissue entrapment. This leads to difficult laryngoscopy. A reduction in the TMJ-incisor distance is associated with a small mandibular length or retrognathia. A reduction in the TMJ-incisor distance is related to a small thyromental distance. Finally, the reduced TMJ-TMJ distance is associated with a narrow palate leading to difficult laryngoscopy. Relative reduction is greater than the volume of the anterior compartment or the upper incisors that require more mandibular protrusion during direct laryngoscopy. The thyromental distance, short mandibular distance, narrow palatal distance, large tongue is also important. Reducing the size of anterior column; micrognathia and Marfan syndrome are could be significant examples. Ludwig's angina, hemorrhage, radiotherapy, macroglossia reduce compliance of anterior column. In these cases, the recommended device type is a videolaryngoscopy with an hyperangulated blade, the use of a straight bladed laryngoscope, which directs the epiglottis, cause narrow shape via compression of anterior column. Depending on the size of the triangular pyramid, a large tongue is often associated with Mallampati III / IV. The presence of the large tongue causes displacing the glottic tissue anteriorly. The most common example can be seen in acromegaly, and pregnancy. Protrusion upper anterior incisors are also associated with difficult laryngoscopy. Despite of the introduction of the laryngoscope blade into the mouth cephalad, the more vertical line of sight, and the shorter mandibular distance, the more prominent upper anterior part require more maxillary protrusion. Leading maxillary incisors are seen when the maxillary incisors are missing and the patient has an upper prosthetic plate. In this case, you need less mandibular protrusion to provide an angle of view during laryngoscopy.
2. The tethering of the Anterior Column may be caused by dysfunction of the TMJ or by calcification of the stylohyoid ligament. TMJ has two functions, sliding movement on maxilla with hinge movement. As a result, TMJ dysfunction leads to limited mouth opening and / or inhibition of mandibular protrusion. Examples of the causes of TMJ dysfunction include bone fractures, spasms and burn contractures that disrupt the joint surface. Calcification of one or both styloid ligaments has been associated with difficult laryngoscopy.

3. Evaluation of submandibular compliance

In the evaluation of submandibular compliance, the previous radiotherapy, neck masses, bleeding or submandibular cavity infection and the presence of severe burns of the neck and jaw should be questioned in the submandibular area. Currently, objective measurement of submandibular compliance as part of the airway assessment still has not been reported. However, there are computerized soft tissue stiffness gauges that measure the strength of the tissue to the constant deformation. These measurements may be advantageous as part of the routine submandibular conformity assessment, but may require further investigation.

\section{Evaluation of Posterior Column}

The evaluation of the range of motion in the atlantooccipital joint (flexion-extension). Rheumatoid arthritis, ankylosing spondylitis, cervical vertebra fusion, manual in-line stabilization or halo limit the mobility of posterior column. In such cases, hyperangulated blade videolaryngoscope and Mc Coy blade are recommended.

\section{Evaluation of Middle Column}

Airway history and imaging tests for airway as X-Ray, CT, Nasopharyngoscopy, Nasal endoscopy, Awake laryngoscopy, Lateral neck X-Ray, CXR, CT neck, MRI neck.

Infections such as abscesses and epiglottitis, foreign bodies, tumours, obstructive sleep apnoea, laryngeal oedema, upper airway burns can distort middle column passage. Appropriate devices; Standard blade videolaryngoscopy, Direct laryngoscopy with a MacIntosh blade, Fiberoptic intubation. 
The Prediction of Difficult Airway: is it Possible?

\section{Airway Evaluation}

There are Conventional airway assessment methods and New airway assessment methods.

\section{Classical Airway Evaluation Tests}

The history of the patient with a history of difficult airway history, facial dysmorphisism, Mouth opening, visibility of oropharyngeal distance, dentition, TMJ mobility, Thyromental distance, Sternomental distance test and the Mallampati score. The actual predictive value of the Mallampati score is approximately 50\%, but class 3 means 8 times higher risk for difficult intubation.

Models such as the Wilson risk score, Arne risk index are being used and have lower sensitivity than Cochrane analysis for predicting difficult airway. The upper lip bite test was found to be the strongest diagnostic test (5).

\section{New Airway Assessment Methods}

Routine airway assessment is largely unreliable in predicting difficult intubation, and despite all preperation we are unexpectedly seen in $90 \%$ of the patients. Recently, ultrasonography is not only an important tool for airway management but also for dynamic airway assessment (6). The role of virtual endoscopy and 3D computed tomographic reconstruction images appears to provide more visualization and difficulty in airway management. New techniques are Nasal endoscopy, Awake laryngoscopy, CT neck, MRI neck, Usg. Suprahyoid and infrahyoid ultrasonographic measurements are used for difficult airway prediction. The measurements at different levels of the anterior neck soft tissue might shed light on difficult airway prediction. The facial analyzes are also useful in increasing predictions by using computer and promising to reduce false negativity. Duggan et al. disseminated their Airway App from 2016 and share their experiences about these front of neck initiatives. Virtual Endoscopy, 3D system, has been used by radiologists for many years to create images of the tracheobronchial tree
VE and to be used for multidimensional imaging for pathological sampling. It provides imaging from the naso-oropharynx to carina.

\section{ConClusion}

We need stronger prediction model to diagnose the difficult airway. Inadequate airway assessment leads to incorrect planning and poor results. An excellent airway assessment test is not yet available. As long as it is integrated into clinical practice to create alternative airway management plans.

\section{REFERENCES}

[1] Cook TM, Woodall N, Frerk C. Major complications of airway man- agement in the UK: results of the 4th National Audit Project of the Royal College of Anaesthetists and the Difficult Airway Society. Part 1 Anaesthesia. Br J Anaesth 2011; 106: 617-31.

[2] Saracoglu A. "Cannot ventilate cannot Intubate" Newborns. Glob J Anesthesiol 2014; 1(1): 28.

[3] Patil VU, Stehling LC, Zauder HL. Predicting the difficulty of intubation utilizing an intubation guide. Anaesthesiology 1983; 10: 32.

[4] Hubert V, Duwat A, Deransy R, Mahjoub Y, Dupont H. Effect of Simulation Training on Compliance with Difficult Airway Management Algorithms, Technical Ability, and Skills Retention for Emergency Cricothyrotomy. Anesthesiology 2014; 120:999-1008.

[5] Roth D, Pace NL, Lee A, Hovhannisyan K, Warenits AM, Arrich J, Herkner H. Airway physical examination tests for detection of difficult airway management in apparently normal adult patients.Cochrane Database Syst Rev. 2018 May 15;5:CD008874.

[6] Falcetta S, Cavallo S, Gabbanelli V, Pelaia P, Sorbello M, Zdravkovic I, Donati A. Evaluation of two neck ultrasound measurements as predictors of difficult direct laryngoscopy: A prospective study. Eur J Anaesthesiol 2018; 35: 605-612.

Citation: Ayten Saracoglu, Kemal Tolga Saracoglu. The Prediction of Difficult Airway: is it Possible?. Archives of Anesthesiology. 2019; 2(1): 32-35

Copyright: (C) 2019 Ayten Saracoglu, Kemal Tolga Saracoglu. This is an open access article distributed under the Creative Commons Attribution License, which permits unrestricted use, distribution, and reproduction in any medium, provided the original work is properly cited. 